\title{
The colorectal cancer-associated faecal microbiome of developing countries resembles that of developed countries
}

Caroline Young $^{1 *}$ (D), Henry M. Wood ${ }^{1}$, Ramakrishnan Ayloor Seshadri ${ }^{2}$, Pham Van Nang ${ }^{3}$, Carlos Vaccaro ${ }^{4}$, Luis Contreras Melendez ${ }^{5}$, Mayilvahanan Bose ${ }^{2}$, Mai Van Doi ${ }^{3}$, Tamara Alejandra Piñero ${ }^{4}$, Camilo Tapia Valladares ${ }^{5}$, Julieta Arguero ${ }^{4}$, Alba Fuentes Balaguer ${ }^{1}$, Kelsey N. Thompson ${ }^{6}$, Yan Yan ${ }^{6}$, Curtis Huttenhower ${ }^{6}$ and Philip Quirke ${ }^{1}$

\begin{abstract}
Background: The incidence of colorectal cancer (CRC) is increasing in developing countries, yet limited research on the CRC- associated microbiota has been conducted in these areas, in part due to scarce resources, facilities, and the difficulty of fresh or frozen stool storage/transport. Here, we aimed (1) to establish a broad representation of diverse developing countries (Argentina, Chile, India, and Vietnam); (2) to validate a 'resource-light' samplecollection protocol translatable in these settings using guaiac faecal occult blood test (gFOBT) cards stored and, importantly, shipped internationally at room temperature; (3) to perform initial profiling of the collective CRCassociated microbiome of these developing countries; and (4) to compare this quantitatively with established CRC biomarkers from developed countries.
\end{abstract}

Methods: We assessed the effect of international storage and transport at room temperature by replicating gFOBT from five UK volunteers, storing two in the UK, and sending replicates to institutes in the four countries. Next, to determine the effect of prolonged UK storage, DNA extraction replicates for a subset of samples were performed up to 252 days apart. To profile the CRC-associated microbiome of developing countries, gFOBT were collected from 41 treatment-naïve CRC patients and 40 non-CRC controls from across the four institutes, and V4 16S rRNA gene sequencing was performed. Finally, we constructed a random forest (RF) model that was trained and tested against existing datasets from developed countries.

Results: The microbiome was stably assayed when samples were stored/transported at room temperature and after prolonged UK storage. Large-scale microbiome structure was separated by country and continent, with a smaller effect from CRC. Importantly, the RF model performed similarly to models trained using external datasets and identified similar taxa of importance (Parvimonas, Peptostreptococcus, Fusobacterium, Alistipes, and Escherichia).

Conclusions: This study demonstrates that gFOBT, stored and transported at room temperature, represents a suitable method of faecal sample collection for amplicon-based microbiome biomarkers in developing countries and suggests a CRC-faecal microbiome association that is consistent between developed and developing countries.

Keywords: Microbiota, gFOBT, Argentina, Chile, India, Vietnam

\footnotetext{
* Correspondence: c.young@leeds.ac.uk

'Pathology \& Data Analytics, Leeds Institute of Medical Research at St James's University Hospital, University of Leeds, Level 4 Wellcome Trust Brenner Building, Leeds LS9 7TF, UK

Full list of author information is available at the end of the article
}

(c) The Author(s). 2021 Open Access This article is licensed under a Creative Commons Attribution 4.0 International License, which permits use, sharing, adaptation, distribution and reproduction in any medium or format, as long as you give appropriate credit to the original author(s) and the source, provide a link to the Creative Commons licence, and indicate if changes were made. The images or other third party material in this article are included in the article's Creative Commons licence, unless indicated otherwise in a credit line to the material. If material is not included in the article's Creative Commons licence and your intended use is not permitted by statutory regulation or exceeds the permitted use, you will need to obtain permission directly from the copyright holder. To view a copy of this licence, visit http://creativecommons.org/licenses/by/4.0/. The Creative Commons Public Domain Dedication waiver (http://creativecommons.org/publicdomain/zero/1.0/) applies to the data made available in this article, unless otherwise stated in a credit line to the data. 


\section{Background}

Colorectal cancer (CRC) is the fourth commonest cause of global cancer-related deaths. Incidence rates have traditionally been highest in developed countries, but are increasing in developing countries, many of which are ill-equipped to respond to this new burden of disease [1]. There is growing evidence of an association between $\mathrm{CRC}$ and an altered faecal microbiome, with the potential to develop novel screening, prognostic or therapeutic markers. Certain bacteria have been proposed as putative oncomicrobes and specific genetic elements such as toxins have been implicated [2]. However, the majority of CRC-microbiome research has profiled developed cohorts. Of the limited number of studies conducted in developing countries, most have been small pilot studies [3-7]. It cannot be assumed that results from developed countries will be generalisable to developing populations, as the health-associated microbiome of developed and developing populations has been shown to differ taxonomically and functionally [8].

One of the biggest limitations to conducting microbiome research in developing countries is storage and transport of frozen stool, which is widely considered the gold standard. Alternative methods have been proposed, and include storing faeces on screening cards (Flinders Technology Associates (FTA) cards or guaiac faecal occult blood test (gFOBT) CRC screening cards) at room temperature [9-15]. Two studies have indicated that cards could be used to store stool at high ambient temperatures, such as those of many developing countries $[16,17]$. However, these studies did not assess the effect of international transport on microbiome stability. Many microbiome studies use samples collected on site or transported frozen, severely limiting protocol options in developing countries. Other protocols have been explored for collection, storage, and transport at ambient temperatures for gut microbiome studies in developing nations, including gFOBT card variants. However, this still requires thorough testing and optimisation for clinical use, particularly as this is not the equivalent of storage in temperature-controlled settings (transport temperatures are likely to be highly variable, encompassing transport at outside-temperature to the airport, transport within the cargo of an aeroplane and transit times of $\sim$ weeks).

To improve the field's ability to conduct low-cost gut microbiome profiling for CRC screening in developing countries, and to provide a pilot assessment of the global CRC-linked microbiome, we established a network comprising researchers from the continents of South America (Argentina and Chile, Development Assistance Committee (DAC)-listed upper-middle income countries at the time of the study), South East Asia (India and Vietnam, DAC-listed lower-middle income countries), and Europe (UK) as a control. These countries represent a range of increasing CRC incidence rates (Age Standardised World Rate in 2018 per 100,000 person-years: India 4.4; Vietnam 13.4; Chile 20.7; Argentina 25.0; UK 32.1) [18-22]. With the exception of the UK, limited microbiome research, in particular CRC-microbiome profiling, has been conducted in these countries $[6,23$, 24] We sought to address these current limitations by assessing whether gFOBT cards collected both in the country of interest and from UK volunteers, then stored/transported at ambient temperatures, could assess CRC-associated microbes comparably around the globe. After establishing the efficacy of our methodology, we compared the faecal microbiomes of ten CRC patients and ten non-CRC controls each from India, Vietnam, Chile, and Argentina, using a standardised methodology to mitigate technical biases, and found the resulting CRC-associated amplicon profiles to be comparable with those from existing CRC-associated metagenomes from developed countries.

\section{Methods \\ UK volunteer samples}

Replicate gFOBT samples were created to investigate the effect of international transport and storage (Fig. 1a). A convenience group of five healthy UK volunteers (relatives of a member of the UK research team) was used; each volunteer donated a stool. Volunteers were aged between 28 and 66, had no history of colonoscopy or antibiotic use within the preceding 6 months, and had no comorbidities. Each stool was used to make ten gFOBT (Hema Screen, Immunostics, Inc). gFOBT were stored at room temperature for $24 \mathrm{~h}$. Developer solution (Hema Screen, Immunostics, Inc) was then applied and gFOBT were left to dry. gFOBT were stored in individual sealed bags at room temperature prior to transit (115-171 days).

Two gFOBT from each volunteer remained within the UK; these acted as baseline samples. Two gFOBT from each volunteer were transported at ambient temperature to India, Vietnam, Chile, and Argentina (transit time 48 days). Of these, one from each volunteer was stored at room temperature for a short period (2-11 days, dependent upon courier collection) before being returned to the UK (transit time 3-6 days). gFOBT were transported at ambient temperature, except for the gFOBT from Argentina which were received with ice packs due to a mistake by the courier. To limit batch effects, DNA extraction was performed upon receipt of samples from all four countries, and DNA extraction of samples which had remained in the UK was performed at the same time.

To assess the effect of the storage conditions which the $\mathrm{CRC} /$ non-CRC control samples had been subject to, 


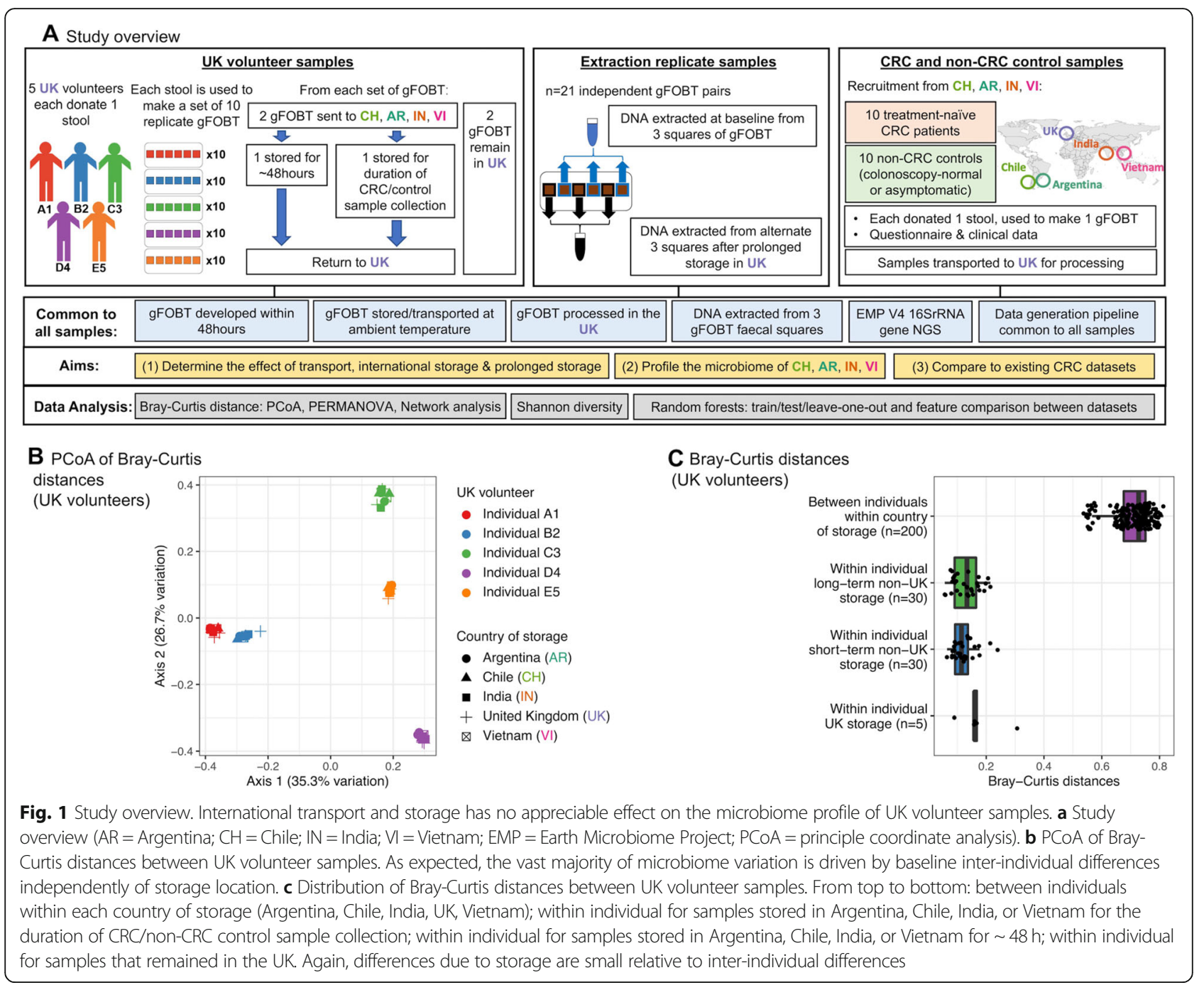

a second set of gFOBT was stored in India, Vietnam, Chile, and Argentina at room temperature for the duration of CRC/non-CRC control sample collection (Vietnam 29 days, Argentina 76 days, India 115 days, and Chile 196 days). The recorded laboratory temperatures were as follows: UK (mean monthly temperature 20$23^{\circ} \mathrm{C}$, maximum $27^{\circ} \mathrm{C}$ ), Vietnam (mean not available, maximum $25^{\circ} \mathrm{C}$ ), Argentina (mean $15^{\circ} \mathrm{C}$, maximum $20^{\circ} \mathrm{C}$ ), Chile (mean $20^{\circ} \mathrm{C}$, maximum $22^{\circ} \mathrm{C}$ ), and India (mean $24^{\circ} \mathrm{C}$, maximum $27^{\circ} \mathrm{C}$ ). Samples were returned at ambient temperature with the CRC/non-CRC control samples (transit time 5-10 days), and DNA extraction was performed upon receipt.

\section{CRC and non-CRC control samples}

gFOBT and clinical data were collected from ten CRC patients and ten non-CRC controls each from Vietnam (Can Tho University of Medicine and Pharmacy, Can Tho, April-June 2018), Argentina (Hospital Italiano de Buenos Aires, June-August 2018), India (Cancer Institute (WIA)
Chennai, July-October 2018), and Chile (Universidad de los Andes Santiago, October 2018-February 2019, samples collected in fact from 11 CRC patients and ten nonCRC controls), to give a total cohort of 81 individuals (Fig. 1a). Non-CRC controls comprised either people with a normal bowel at colonoscopy (Vietnam and Argentina) or asymptomatic individuals working in the affiliated University (Chile and India). Both control groups (colonoscopynormal controls or asymptomatic controls) are variously used in microbiome studies; we offered flexibility as the institutes in Chile and India had limited access to colonoscopy-normal controls (colonoscopy is less readily available in some developing countries). Colonoscopies were carried out a minimum of 2 weeks prior to sample collection (during which time the majority of taxa return to baseline). CRC patients were treatment-naïve. Study participants were aged over 18. Exclusion criteria included the following: antibiotic use within the preceding 6 months; foreign travel within the preceding 2 weeks; colonoscopy within the preceding 2 weeks; related to another 
study participant; colostomy; history of previous CRC/adenoma, colorectal surgery, pelvic radiation, or chemotherapy; known CRC syndrome or family history of hereditary CRC; and coexistent IBD or infectious bowel disease.

To limit batch effects, samples from CRC patients and non-CRC controls were collected as far as possible alternately and were transported and processed as a single batch. Participants provided a stool which was used to prepare a gFOBT. Developer solution was applied the same day in the majority of instances (occasionally the following day), and gFOBT were left to dry. gFOBT were stored in individual sealed bags at room temperature. Once collection was complete, samples were returned to the UK at ambient temperature.

\section{DNA extraction replicate samples}

To assess the effect of prolonged UK storage at room temperature, DNA extraction replicates were created from a subset of the CRC/non-CRC control samples ( $n=21$ pairs), by extracting DNA from three squares of faecally loaded card at baseline (details below) and subsequently from the three remaining squares after prolonged UK storage (Fig. 1a). Time between DNA extraction of replicate pairs was as follows: Chilean samples 27 days, Indian samples 140 days, Argentinian samples 211 days, and Vietnamese samples 252 days.

\section{DNA extraction}

Sample processing was performed at the University of Leeds. The QIAamp DNA Stool Mini Kit (Qiagen, Germany) was used until its discontinuation in August 2018, whereupon the equivalent QIAamp DNA Mini Kit (Qiagen, Germany) and Buffer ASL (Qiagen, Germany) were used.

From each gFOBT, three squares of faecally loaded card were dissected and processed as a single combined sample. The rationale of this approach is that it subsamples a larger volume of stool, ensuring adequate biomass even from thinly smeared gFOBT (the volume of stool per gFOBT can be very variable when prepared by study participants), and leaves three squares remaining for alternative analysis or DNA extraction replicates. Next, $800 \mu \mathrm{l}$ of Buffer ASL was added and samples were incubated at $23^{\circ} \mathrm{C}$ on a Thermomixer Comfort (Eppendorf $\mathrm{UK})$ at $850 \mathrm{rpm}$ for $1 \mathrm{~h}$. Samples were briefly centrifuged and supernatant transferred to pathogen lysis tubes (S) (Qiagen, Germany). Samples were agitated (Vibrax VXR, IKA, UK) at a motor setting of $1800-2200$ for $10 \mathrm{~min}$ followed by incubation at $95^{\circ} \mathrm{C}$ on the Thermomixer at $850 \mathrm{rpm}$ for $15 \mathrm{~min}$. Samples were then centrifuged at $18625 \mathrm{~g}$ for $1 \mathrm{~min}$, and supernatant was transferred to a tube containing $173 \mu \mathrm{l}$ of $10 \mathrm{M}$ ammonium acetate. We then vortexed the samples and placed them on ice for $5 \mathrm{~min}$, then centrifuged at $18625 \mathrm{~g}$ for $5 \mathrm{~min}$.
Supernatant was transferred to a tube containing $725 \mu \mathrm{l}$ of propan-2-ol, vortexed, and placed on ice for $30 \mathrm{~min}$. Then, samples were centrifuged at $18625 \mathrm{~g}$ for $10 \mathrm{~min}$, supernatant was discarded, and $1 \mathrm{ml}$ of $70 \%$ ethanol was added. Samples were centrifuged at $18625 \mathrm{~g}$ for $5 \mathrm{~min}$, supernatant discarded, and $500 \mu \mathrm{l} 70 \%$ ethanol was added. Samples were centrifuged at $18625 \mathrm{~g}$ for $3 \mathrm{~min}$, supernatant discarded, and the samples left for $10 \mathrm{~min}$ to evaporate residual ethanol. Two hundred microliters of tris-EDTA was added, and after $10 \mathrm{~min}$, samples were vortexed and added to tubes containing $200 \mu \mathrm{l}$ of Buffer AL (QIAamp DNA Mini Kit). Fifteen microliters of Proteinase K (QIAamp DNA Mini Kit) was added; the samples were vortexed and incubated at $70^{\circ} \mathrm{C}$ on the Thermomixer at $650 \mathrm{rpm}$ for $10 \mathrm{~min}$. The QIAamp DNA Mini Kit protocol was then followed. To elute DNA, $100 \mu \mathrm{l}$ of UV-irradiated molecular biology grade water was added to samples for 5 min before centrifuging at $18625 \mathrm{~g}$ for $1 \mathrm{~min}$.

\section{S rRNA library preparation and NGS}

The Earth Microbiome Project (EMP) 16S Illumina Amplicon library preparation methodology was followed [25], with Illumina $16 \mathrm{~S}$ V4 primer constructs $515 \mathrm{~F}$ (Parada)-806R (Apprill) [26, 27]. Single (rather than triplicate) PCR reactions were performed per sample, each with a starting amount of $20 \mathrm{ng}$ DNA. One hundred fifty-two samples were sequenced as part of a total pool of 996 samples from other projects, on one lane of an Illumina HiSeq 3000 , for $2 x 150 b p$ sequencing, with a 10 bp index read.

\section{Bioinformatic processing and statistical analysis}

Reads were stripped of adaptors using cutadapt [28]. Further processing was carried out in QIIME2 (version 2019.4) [29]. Reads were trimmed to a maximum of 145 bp, pairs merged, denoised, and representative sequences chosen using DADA2 [30].

Taxa were assigned to representative sequences by the QIIME2 feature classifier using the BLAST+ algorithm [31, 32], aligning sequences against the SILVA version 132 99\% similarity database [33].

Within the QIIME2 environment, samples were rarified to the depth of the sample with fewest QC-passed sequences $(51,000)$, and Shannon index alpha diversity was calculated [34], with significance assessed by the Kruskal-Wallis test [35]. Rarified samples were used to calculate Bray-Curtis beta diversity [36], and principle coordinate analysis (PCoA) was performed. Taxa, representative sequences, and distance matrices were exported from QIIME2 for analysis and graphical representation using $R$ (version 3.5.1).

Significance of differences in beta diversity between groups was assessed by PERMANOVA analysis of Bray- 
Curtis distances performed using the adonis function within vegan [37]. Where necessary, multivariate models were built using repeated measures aware permutations within the PERMANOVA test, to account for repeated measures per individual (https://bitbucket.org/biobakery/ hmp2_analysis/src/default/overview/src/omnibus_tests.r) [38]. Network analysis of genus level Bray-Curtis distances was performed using phyloseq [39].

To investigate the discriminatory performance of the microbiome, a random forest (RF) model [40] was built using the combined cohort, using the packages randomForest [41] and pROC [42]. To adjust for the effects of age, after per-sample normalisation, a linear model of each taxon with age was calculated, and the residual values rather than taxon abundance used to construct a RF model.

Taxa from the combined cohort were compared to other CRC faecal metagenomic datasets [23, 43-45], processed using MetaPhlAn version 3.0 [46]. The datasets contain samples from the following countries: Feng - Austria $(n=107)$; Gupta - India $(n=60)$; Thomas_a and Thomas_ b - Italy $(n=106)$; Vogtmann - USA $(n=104) ;$ Wirbel Germany $(n=125)$; Yachida - Japan $(n=518) ; \mathrm{Yu}-$ Hong Kong, China $(n=128)$; and Zeller - France $(n=114)$. All datasets were collapsed to genus level for comparison with 16S rRNA gene amplicon data. The Thomas_c [44] dataset was merged with the Yachida [45] dataset, as both originated from the same cohort. A random forest (RF) model [40] was built from each dataset using the randomForest [41] and compared with every other dataset using pROC [42]. Area under the curve (AUC) for each validation was recorded, as were taxa importance ranks for each model. For self-vs-self comparisons, each study was randomly split into equal sized training and validation sets 20 times, and mean AUC recorded. Additionally, a leaveone-dataset-out (LODO) comparison was performed, whereby models were built from all but one dataset, and validated on the missing dataset. Finally, taxa differing significantly between groups were obtained using LEfSe (Linear discriminant analysis Effect Size) [47].

Mann-Whitney and Fisher's exact test were performed to assess intra-country demographic differences; hypotheses were two-tailed with a significance level of 0.05 . KruskalWallis ANOVA was performed to assess inter-country differences of tumour size, and post-hoc Dunn $p$ values with Benjamini-Hochberg FDR adjustment were calculated.

The dataset supporting the conclusions of this article is available in the ENA repository [48]: https://www.ebi. ac.uk/ena/data/view/PRJEB36789

\section{Results}

CRC and non-CRC control populations and gFOBT-based microbiome profiling strategies

In total, we profiled 16S rRNA gene amplicons from stool representing the gut microbiomes of 41 CRC participants and 40 non-CRC controls, spanning Argentina, Chile, India, and Vietnam (Fig. 1, Table 1). The majority of tumours were located in the caecum/ascending colon or sigmoid/rectum and were stage pT3 or pT4 (Table 2).

Age is, of course, a substantial contributor to CRC development, although it is not generally a major driver of microbiome variation within the range studied here (e.g. associated with $\sim 5 \%$ of taxonomic variation, see below). The median age of non-CRC controls from Argentina, Chile, and India was substantially younger than the corresponding CRC patients, and the median age of CRC patients from India and Vietnam was younger than that of CRC patients from Chile and Argentina (Table 1). While this did not substantially affect subsequent analyses when tested, we note it both here and in our initial profiles of microbiome composition below. Non-CRC controls from Chile and India were asymptomatic individuals working in the affiliated Universities. Non-CRC controls from Vietnam and Argentina underwent colonoscopy, yielding descriptions of 'macroscopically normal bowel' $(n=14)$, diverticulosis $(n=4)$, or 'macroscopically normal bowel with haemorrhoids' $(n=2)$, which are grouped together in subsequent analyses as non-CRC control (it should be noted that these are common colonoscopy findings in older populations and have not been associated with a distinct microbiome profile) [49] The total reads/sample (CRC patients, non-CRC controls and UK volunteers) were 51,000-167,000 (median $117,000)$.

\section{International transport and storage of gFOBT, and prolonged storage in the UK, has no appreciable effect on results}

To first investigate the effect of international transport and storage, 50 replicate gFOBT were created using stool from a subset of the total population, comprising five UK volunteers (ten replicate gFOBT/volunteer). Two gFOBT from each volunteer remained in the UK. Two gFOBT from each volunteer were transported to institutes in Argentina, Chile, India, and Vietnam; of these, one from each volunteer was stored for a short duration, and one was stored for the duration of $\mathrm{CRC} /$ non-CRC control sample collection (Fig. 1a).

Neither country nor duration of storage had a significant effect on the microbiome structure of the UK volunteer samples, which as expected grouped by UK volunteer (Fig. 1b, c, Additional file 1: Fig. S1A). PERM ANOVA of Bray-Curtis dissimilarity confirmed this, quantifying the effect of UK volunteer as $R^{2}=94 \%$ ( $p=$ 0.001) (Fig. 2b, Additional file 1: Table S1). Each UK volunteer's taxonomic composition was assessed essentially equivalently across the different storage methods, and whilst there was a minor amount of taxonomic 
Table 1 CRC and non-CRC control characteristics

\begin{tabular}{|c|c|c|c|c|c|c|c|c|}
\hline & \multicolumn{2}{|l|}{ Argentina } & \multicolumn{2}{|l|}{ Chile } & \multicolumn{2}{|l|}{ India } & \multicolumn{2}{|l|}{ Vietnam } \\
\hline & CRC & NC & CRC & NC & CRC & NC & CRC & NC \\
\hline Number of participants & 10 & 10 & 11 & 10 & 10 & 10 & 10 & 10 \\
\hline Male & 4 & 6 & 4 & 4 & 7 & 6 & 5 & 3 \\
\hline \multirow[t]{2}{*}{ Median age (range) } & $81(61-89)$ & $55.5(37-72)$ & $70(56-86)$ & $34(22-75)$ & $56.5(33-73)$ & $34(26-45)$ & $58(49-88)$ & $58.5(37-71)$ \\
\hline & \multicolumn{2}{|c|}{$M W p=4.4 \times 10^{-4}$} & \multicolumn{2}{|c|}{ MW $p=3.5 \times 10^{-3}$} & \multicolumn{2}{|c|}{$M W p=1 \times 10^{-3}$} & & \\
\hline \multirow[t]{2}{*}{ History of colonoscopy ${ }^{a}$} & 10 & 10 & 11 & 0 & 8 & 0 & 10 & 10 \\
\hline & & & \multicolumn{2}{|c|}{ FE $p<1 \times 10^{-5}$} & \multicolumn{2}{|c|}{ FE $p=7 \times 10^{-4}$} & & \\
\hline \multirow[t]{2}{*}{ Medication use } & 9 & 6 & 9 & 3 & 6 & 1 & 10 & 10 \\
\hline & & & \multicolumn{2}{|c|}{ FE $p=3 \times 10^{-2}$} & & & & \\
\hline \multirow[t]{2}{*}{ Comorbidities $^{\mathrm{b}}$} & 8 & 4 & 9 & 5 & 7 & 0 & 3 & 3 \\
\hline & & & & & \multicolumn{2}{|c|}{$\mathrm{FE} p=3.1 \times 10^{-3}$} & & \\
\hline Current smoker $^{c}$ & 0 & 1 & 0 & 3 & 1 & 0 & 3 & 1 \\
\hline \multirow[t]{2}{*}{ Drinks alcohol } & 3 & 4 & 4 & 9 & 1 & 3 & 3 & 2 \\
\hline & & & \multicolumn{2}{|c|}{ FE $p=2.4 \times 10^{-2}$} & & & & \\
\hline Vegetarian & 0 & 0 & 0 & 0 & 2 & 6 & 0 & 1 \\
\hline
\end{tabular}

CRC CRC patient, NC non-CRC control

Mann-Whitney (MW) and Fisher's exact test (FE) were performed to assess intra-country differences; hypotheses were two-tailed with a significance level of 0.05 . Significant differences are in bold and the $p$ value stated

a History of colonoscopy indicates whether participants had ever had a colonoscopy with bowel preparation prior to sample collection

${ }^{b}$ Comorbidities included in our population profile, but not as substantial analysis covariates: hypertension, gastric ulcer, gastro-oesophageal reflux disease, insulin resistance/diabetes, thyroid disease, obesity, and hypercholesterolaemia

${ }^{c}$ Current smoker includes participants who stopped smoking within the preceding month

Table 2 Tumour characteristics

\begin{tabular}{|c|c|c|c|c|}
\hline & Argentina $n=10$ & Chile $n=11$ & India $n=10$ & Vietnam $n=10$ \\
\hline \multicolumn{5}{|l|}{ Tumour location ${ }^{a}$} \\
\hline Caecum or ascending colon & 5 & 5 & 2 & 0 \\
\hline Transverse colon & 0 & 0 & 0 & 1 \\
\hline Descending colon & 0 & 0 & 0 & 1 \\
\hline Sigmoid colon or rectum & 4 & 6 & 8 & 8 \\
\hline Data not available & 1 & 0 & 0 & 0 \\
\hline \multicolumn{5}{|c|}{ Maximum tumour size in one direction } \\
\hline Median $(\mathrm{cm})$ (range) & $4(0.7-7)$ & $6(2.5-11)$ & $5(3-5.5)$ & $4(3-5)$ \\
\hline Data not available & 2 & 0 & 7 & 0 \\
\hline \multicolumn{5}{|c|}{$\begin{array}{l}\text { Kruskal Wallis ANOVA } p=2.9 \times 10^{-2} \text { Post-hoc Dunn significant pairwise differences (Benjamini-Hochberg FDR adjusted): Chile vs } \\
\text { Vietnam } p=3.5 \times 10^{-2}\end{array}$} \\
\hline \multicolumn{5}{|c|}{ Tumour stage (TNM8) ${ }^{\mathrm{a}}$} \\
\hline $\mathrm{T} 1$ & 0 & 2 & 0 & 0 \\
\hline $\mathrm{T} 2$ & 1 & 1 & 4 & 0 \\
\hline T3 & 8 & 8 & 4 & 0 \\
\hline T4 & 0 & 0 & 0 & 10 \\
\hline Data not available & 1 & 0 & 2 & 0 \\
\hline
\end{tabular}

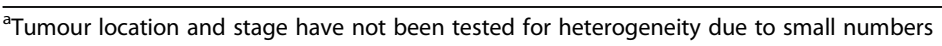




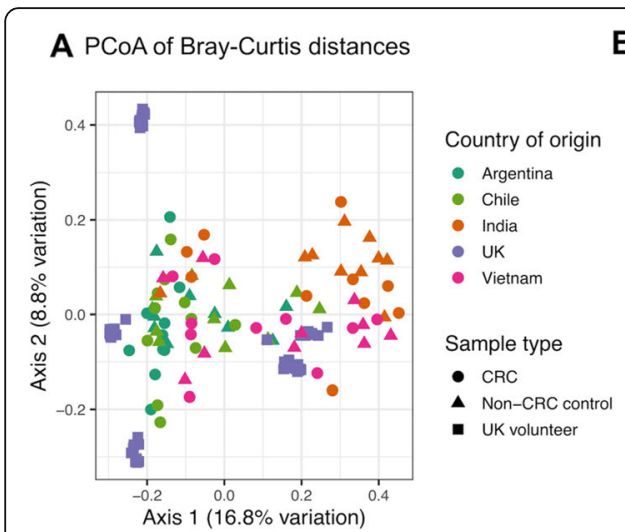

D Shannon diversity (CRC/non-CRC controls)

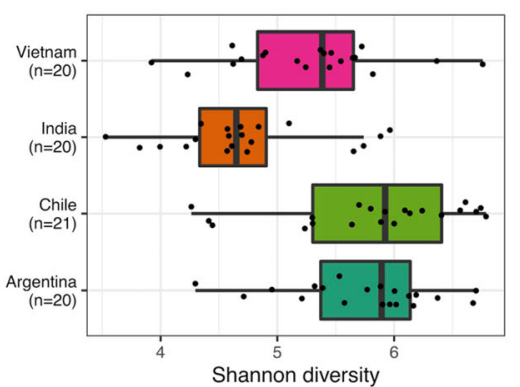

B Bray-Curtis distance PERMANOVA
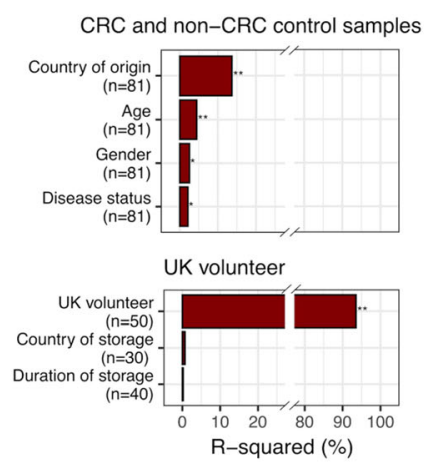

C Bray-Curtis distances (CRC/non-CRC controls)

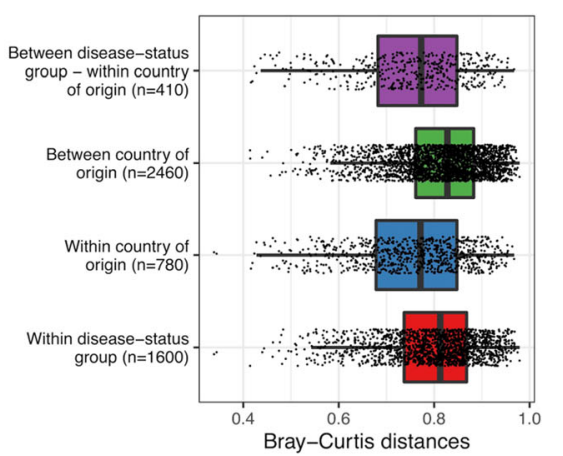

E Bray-Curtis distance network analysis

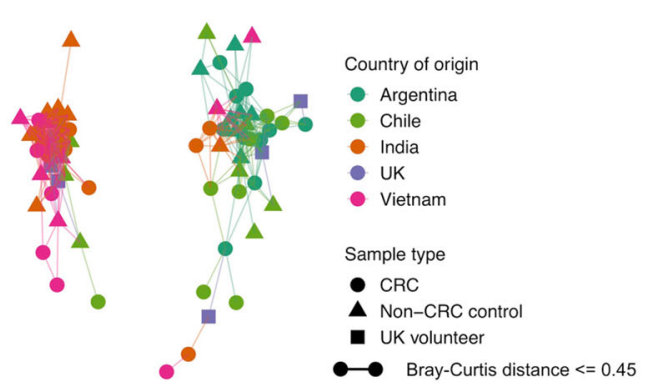

Fig. 2 Country and continent drive microbiome structure. a PCOA of Bray-Curtis distances between all samples. b PERMANOVA based on Bray-Curtis distances between CRC and non-CRC control samples and UK volunteer samples. For the CRC/non-CRC control samples: 'country of origin' = Argentina, Chile, India or Vietnam; 'disease status' $=$ CRC or non-CRC control. For the UK volunteer samples: 'country of storage' $=$ Argentina, Chile, India, Vietnam or UK (only samples stored in the UK or short-term outside the UK were used in this analysis, as these samples underwent DNA extraction at the same time); 'duration of storage' $=$ short-term or long-term storage outside the UK (samples which remained in the UK were excluded from this analysis). ${ }^{* *} p \leq 0.01 .{ }^{*} p \leq 0.05$. $\mathbf{c}$ Distribution of Bray-Curtis distances for CRC and non-CRC control samples: 'disease-status group' $=$ CRC or non-CRC control. $\mathbf{d}$ Shannon diversity indices for CRC and non-CRC control samples. At the taxonomic resolution achievable from the data, Asian individuals generally had lower diversity than South American individuals. e Network representation of all samples based on Bray-Curtis distances. Each UK volunteer is represented by a single point, reflecting the average relative abundance across all samples derived from that individual. Overall dissimilarities are driven by a weak segregation between Asian and South American microbiomes

variability (average inter-individual replicate BrayCurtis dissimilarity 0.13), this affected both samples which remained in the UK and samples which were transported and stored internationally (Additional file 1: Fig. S1B).

To determine whether the microbiome would remain stable if samples were stored for a prolonged period at room temperature in the UK pending DNA extraction, extraction replicates were created from a subset of the CRC/non-CRC control samples (Fig. 1a). DNA extraction was performed upon sample receipt, by dissecting three squares of faecally loaded card, and extraction replicates were created by dissecting the alternate three squares after a period of storage at room temperature. Pairs of replicate samples had similar microbiome structures and taxonomic profiles (average inter-individual replicate Bray-Curtis dissimilarity $=0.17$ ) (Additional file 1: Fig. S2A-C). No significant taxonomic differences were detected by LEfSe between the groups of baseline and replicate samples.

\section{Geography drives CRC-independent gut microbiome structure of participants}

Aside from inter-individual differences, the greatest determinant of microbiome structure was country of origin (Fig. 2a). PERMANOVA quantified this based on BrayCurtis distances $\left(R^{2}=14 \%\right)(p=0.001)$, with 'disease status' (CRC or non-CRC control) contributing far less $\left(R^{2}=2 \%\right)(p=0.019)$ (Fig. 2b, c, Additional file 1: Table S1). A significant difference in alpha diversity (Shannon) was likewise detected between countries (Kruskal Wallis $p=4 \times 10^{-5}$ ). Specifically, the alpha diversities of Vietnamese and Indian samples were significantly lower than those of the Argentinian and Chilean samples, and those of the Indian samples were lower than the Vietnamese (Fig. 2d). No significant difference in Shannon diversity index was detected between overall CRC and non-CRC control samples (Kruskal Wallis $p=0.28$ ).

In addition to country of origin, continent was itself a driver of microbiome structure, with the majority of the Asian samples (India and Vietnam) distinct from the 
majority of the South American samples (Chile and Argentina) (Fig. 2e). In our study, similar to previous reports, Asian samples had a significantly higher relative abundance of Prevotella (LDA score 5.173, $p=2.79 \times$ $10^{-6}$ ) and lower relative abundance of Bacteroides (LDA score 4.841, $\left.p=2.32 \times 10^{-6}\right)$ compared with South American samples (Additional file 1: Fig. S3A-C).

\section{The CRC-associated microbiome of developing countries resembles that of developed countries}

To determine the potential of the microbiome to discriminate between CRC and non-CRC control samples, a RF model was built using the combined total dataset of CRC and non-CRC controls (AUC 0.77 (CI 0.67-0.87)). Given the age-imbalance within our dataset, we compared the result with an age-adjusted RF model; the age-adjusted RF model performed equivalently (AUC 0.80 (CI 0.69-0.89)), confirming that age does not account for the discriminatory performance of the RF model.

Next, the combined total dataset of CRC and nonCRC control amplicon profiles was compared to CRC faecal shotgun metagenomic datasets from the existing literature (Fig. 3) [23, 43-45] . These nine existing studies included 1262 total samples from the following countries: France $(n=114)$, Austria $(n=107)$, Italy $(n=106)$,
Germany $(n=125)$, USA $(n=104)$, India $(n=60)$, Hong Kong, and China $(n=128)$ and Japan $(n=518)$. A random forest (RF) model trained using the current study's amplicon dataset and tested using the external metagenomic datasets, paralleling previous methodology [44], performed strikingly similarly (mean AUC 0.75) to models trained using the external metagenomes (mean AUC 0.71-0.80) (Fig. 3a). Similarly, models trained using the external datasets performed similarly when tested using the current study's dataset (mean AUC 0.78, leaveone-dataset-out AUC 0.85 ) or the other external datasets (mean AUC 0.67-0.85, leave-one-dataset-out AUC 0.73-0.91).

RF models built using each dataset ranked CRCassociated taxa of greatest discriminatory importance similarly (Fig. 3b, Additional file 1: Fig. S4A). The five CRC-associated taxa which were of greatest importance to the majority of the models (Parvimonas, Peptostreptococcus, Fusobacterium, Alistipes, and Escherichia) were ranked in the top ten taxa by the model built using the current study's dataset. The five most important taxa for the current study's model were Peptostreptococcus, Odoribacter, Parvimonas, Porphyromonas, and Alistipes. As mentioned, Peptostreptococcus, Parvimonas, and Alistipes were ranked highly by the majority of external

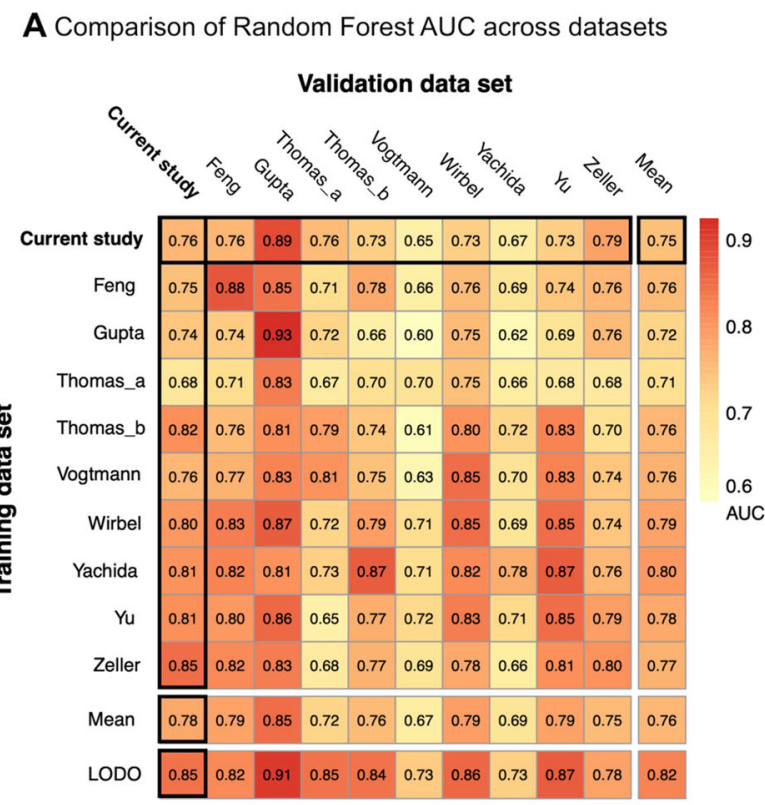

B Random Forest feature ranking

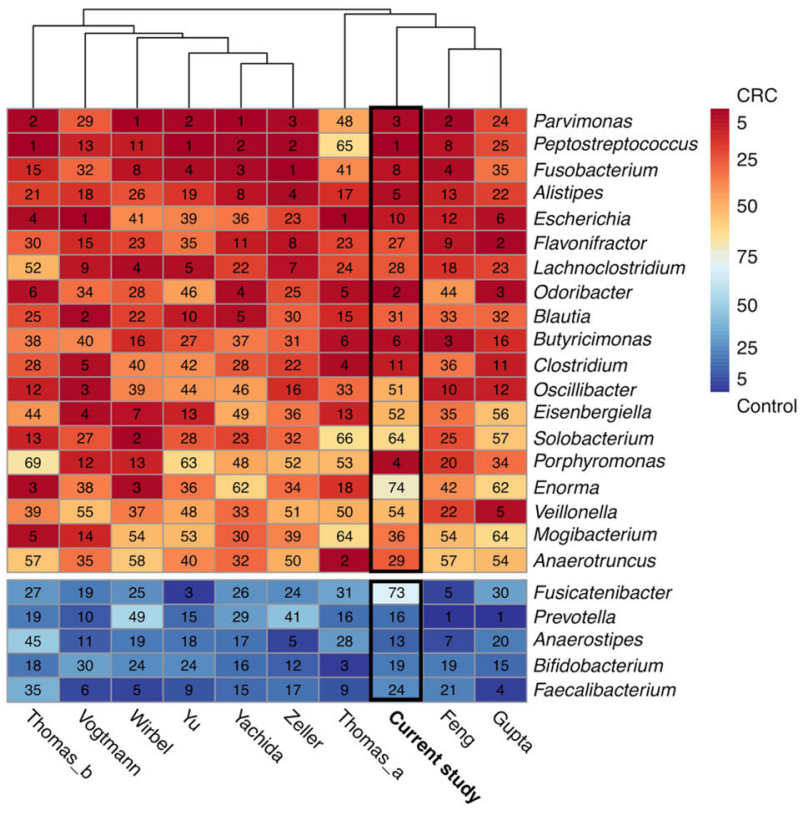

Fig. 3 CRC prediction performance is comparable and taxonomically similar across datasets. a Cross-prediction matrix demonstrating random forest AUC based on genus-level relative abundances. LODO (leave-one-dataset-out) refers to the AUC generated by training a model using all but the dataset of the associated column and testing it using the dataset of that column. The datasets contain samples from the following countries: Feng - Austria; Gupta - India; Thomas_a - Italy; Thomas_b - Italy; Vogtmann - USA; Wirbel - Germany; Yachida - Japan; Yu - Hong Kong, China; Zeller - France. $\mathbf{b}$ The importance of each genus for the cross-validation performance in each dataset using gini values. Only genera in the top five in at least one dataset are shown 
models. In contrast, Odoribacter and Porphyromonas were ranked highly by some but not all of the external models; this discrepancy could be secondary to demographic or technical differences between cohorts. Within the current study, CRC-associated taxa differed by country; Peptostreptococcus and Parvimonas were $\mathrm{CRC}$-enriched in cohorts from three of the four countries (India, Chile and Vietnam) (Additional file 1: Fig. S4B-F).

These results together indicate that the CRC-associated microbiome is substantially consistent, and can be consistently measured, across multiple very different international populations. This was true across cohorts and sample handling within this study and surprisingly also proved to hold when comparing these diverse populations to external CRC cohorts. In general, control-associated taxa were of lesser importance to the RF models than CRC-associated taxa. This is likely due to the heterogeneous nature of the current study's control group and of different "healthy" populations' gut microbiomes generally. It suggests that the commonality of some CRCassociated microbiome shifts may be particularly important, either causally or in response to cancer-induced changes in the intestinal microenvironment.

\section{Discussion}

To date, limited microbiome research has been conducted in developing countries, leaving a gap in our knowledge of gut microbial relationships with chronic disease in global populations. We sought to address this in CRC by establishing a network of researchers and participants from Argentina, Chile, India, and Vietnam. First, we demonstrated that gFOBT, stored at room temperature and, importantly, also transported at ambient temperature, were suitable for genus-level faecal microbial taxonomic profiling in a translational setting across these multinational cohorts. This also remained true for gFOBT samples with prolonged storage periods at room temperature in the UK. This allowed us to further profile the faecal microbiomes of CRC patients and non-CRC controls from Argentina, Chile, India, and Vietnam, and, through comparison with external datasets, to demonstrate that the CRC-associated microbiome of these developing countries resembles that of developed countries.

As in previous methodologically similar studies in European or North American populations, the microbiome profile of replicate gFOBT samples from five UK volunteers that were either stored in the UK, or transported and stored abroad (maximum temperature $27^{\circ} \mathrm{C}$ ) for either a short or long (maximum 196 days) duration, was predominantly shaped by inter-individual differences and not appreciably by storage conditions. Similarly, DNA extraction replicate pairs demonstrated consistent microbiome profiles after UK room temperature storage (maximum 252 days). Together, these results indicate that sample analysis did not suffer due to bacterial overgrowth or DNA degradation, despite prolonged storage and transportation at ambient temperature. This is presumably due to the action of Hema Screen developer solution (which contains a stabilised mixture of hydrogen peroxide $(<6 \%)$ and $75 \%$ denatured ethyl alcohol in aqueous solution); notably, developer would not normally be added to screening gFOBT samples immediately upon collection, although a rapid fixation process is easily applicable in field settings. Although not formally assessed by this study, it is expected that an equivalent alcohol/hydrogen-peroxide based solution could be readily made in-house at lowcost, facilitating microbiome-research in developing countries.

Our results agree with and build on existing studies which have also demonstrated the suitability of gFOBT for microbial community amplicon profiling, aiming to validate such methods not only for research but for field clinical settings [9-15]. Our study adds to the small number which have assessed stability after prolonged storage, by demonstrating stability after eight months for 21 replicate pairs. Two studies investigated the microbiome of card samples at higher ambient temperatures; one showed stability of FTA at $4-40{ }^{\circ} \mathrm{C}$ [16] and the other showed stability of gFOBT stored for four days at ambient temperature in Bangladesh [17]. Our study corroborates these findings; additionally, we show that prolonged storage at room temperature in developing countries and international transport of samples at ambient temperature has no detrimental effect. The latter in particular is an important finding, offering an alternative to fresh and/or frozen samples that is practical for use at population scale in international clinical settings at reduced cost. While the types and detail of microbiome profiles that can be obtained from such samples remains limited, it is conversely appropriate for some important population-scale applications such as cancer biomarker testing. We hope these findings will thus encourage others to consider using gFOBT for appropriate epidemiology in settings where more detailed sample types are infeasible.

As expected, we found that for the CRC and non-CRC control samples, the greatest determinant of microbiome structure, aside from inter-individual variation, was country of origin, corresponding to $14 \%$ of overall microbial variation. This is in agreement with a previous study, in which country of origin accounted for a similar amount of variation $\left(R^{2}=22 \%\right)$ [50]. Additionally, countries within continents also showed greater microbial similarity $[51,52]$. Inter-continent differences in microbiome structure were likely a consequence of the inverse 
Prevotella to Bacteroides ratio of Asian and South American samples. Similar results have been previously documented by microbiome studies of healthy Indians, Argentinians, and Chileans and are in keeping with expected differences between these countries [52-58]. The fact that the Asian samples had lower alpha diversities was somewhat surprising. However, other studies have also demonstrated low alpha diversity of faecal samples from healthy Indians, perhaps due to the high relative abundance of Prevotella [54, 56], as clades such as these and Bacteroides can be difficult to distinguish in amplicon-based profiling (thus leading to lower apparent diversity when dominant).

By comparing a random forest model built from our dataset with models built using external, largely developed cohorts, we demonstrated a surprising commonality of the CRC-associated microbiome, particularly CRCassociated taxa, between developed and developing populations. This finding is remarkable in light of technical differences between the studies (method of sample collection and amplicon versus metagenomic sequencing, to name the largest) and the fact that we combined CRC patients and non-CRC controls from four developing countries with distinct microbiome profiles. It should be noted, however, that whilst the direction of the effect is similar between our study and larger, more homogeneous, later-stage CRC cohorts, the magnitude of the effect is understandably smaller. The five taxa which were of greatest importance to the majority of these models were Parvimonas, Peptostreptococcus, Fusobacterium, Alistipes, and Escherichia. Although we were limited in the precision with which we could measure them, within these genera previous studies have associated several of their species with CRC: Peptostreptococcus stomatis [23], Peptostreptococcus anaerobius [45], Fusobacterium nucleatum [59], Parvimonas micra [59], Alistipes finegoldii [59], and pks+ Escherichia coli [2]. Of these, Fusobacterium nucleatum and pks+ Escherichia coli in particular have been suggested as putative 'oncomicrobes'. Fusobacterium nucleatum has been shown to promote tumour proliferation, pro-tumour inflammation and to subvert anti-tumour immune responses [60], whilst colibactin, produced by pks+ Escherichia coli, has been shown to cause DNA damage [2].

Outside of these examples, many of the additional CRC-enriched taxa are oral bacteria that rarely colonise the gut during 'health' but have been implicated in a variety of inflammatory and dysbiotic conditions [61]. It has been hypothesised that oral microbial growth in the colon can cause increased mucosal permeability, with subsequent bacterial invasion, inflammation, and epithelial proliferation, and indeed associated biofilms have been shown to induce tumourigenesis in a mouse model [62-64]. Whether cause or consequence of tumour formation, the fact that these bacteria are found in both developed and developing cohorts points towards the oral microbiome as a shared source of CRC-associated taxa. Geographical differences of the oral microbiome have been described, but the universality of CRCassociated taxa derived from the oral microbiome has not, to our knowledge, been extensively investigated [65].

Continuing to explore the global effects of the microbiome on CRC has the potential to improve both the disease's management worldwide and our understanding of the underlying basic biology. It will be important to expand the cohort by sampling a larger number of participants with more rigorous age and gender matching, in addition to expanding the number of countries profiled, as well as the geographical catchment within countries, many of which show great intra-country diversity. Importantly, microbiome profiling may provide valuable insight into the rising incidence of CRC within these countries, and the shared CRC-associated microbiome raises the potential of a generalisable microbiome-based CRC screening test. To this end, we have demonstrated that gFOBT is a suitable method of faecal sample collection for 16S rRNA gene research in developing countries (Argentina, Chile, India, and Vietnam) and that their CRC-associated microbiome shares many features with that of developed countries. We encourage other researchers to investigate the CRC microbiome in greater depth and in additional populations, with the goal of preventing or treating the disease around the globe.

\section{Conclusions}

Limited CRC-microbiome research has been conducted in developing countries, yet CRC incidence is increasing in these areas. One of the impediments to creating and applying CRC-microbiome biomarkers is the collection of frozen stool samples. Here, we investigated the efficacy of stool-based biomarkers using bowel cancer screening cards (gFOBT), stored and, importantly, transported at room temperature. We then used this technique to investigate the microbiome of CRC patients and controls from four developing countries (Argentina, Chile, India, and Vietnam). Remarkably, we show that the CRC-associated microbiome of these developing countries resembles that of developed countries, even when using limited, field-appropriate, and scalable sampling methods.

\section{Supplementary Information}

The online version contains supplementary material available at https://doi. org/10.1186/s13073-021-00844-8.

Additional file 1: Table S1. PERMANOVA analysis. $P$-values $<0.05$ are shaded grey. $R^{2}$ values are recorded to two decimal places. Fig. S1A. 
Distribution of Bray-Curtis distances between UK volunteer samples. The five UK volunteers are labelled A1-E5. Within individual Bray-Curtis distances are low, despite differences in sample storage. Fig. S1B. Genuslevel taxonomic profile of UK volunteer samples. Each bar represents a sample labelled as: UK volunteer (A1-E5); country of storage $(A R=$ Argentina; $\mathrm{CH}=$ Chile; $\mathrm{IN}=$ India; $\mathrm{VI}=$ Vietnam; UK); storage duration ( $\mathrm{S}=$ short-term storage; $\mathrm{L}=$ long-term storage (i.e. the duration of $\mathrm{CRC} /$ nonCRC control sample collection); $R=$ samples which remained in the UK). The key contains the top 20 taxa (where a genus was described as family_group, groups were merged and only the family name is included for brevity); additional taxa are coloured grey. There is minimal taxonomic variability between samples from the same individual, and taxonomic variability affects both samples which remained in the UK and samples which were transported and stored internationally. Fig. S2A. PCoA of Bray-Curtis distances for extraction replicates. Points are coloured as extraction replicate pairs. Fig. S2B. Distribution of Bray-Curtis distances between extraction replicate samples. Fig. $\mathbf{S 2 C}$. Genus-level taxonomic profile of extraction replicate samples. Each bar represents a sample labelled as follows: country of origin ( $\mathrm{AR}=$ Argentina; $\mathrm{CH}=\mathrm{Chile;} \mathrm{IN}=$ India; $\mathrm{Vl}=$ Vietnam); disease status ( $\mathrm{CRC}=\mathrm{CRC} ; \mathrm{NC}=$ non-CRC control); sample ID; whether the sample is an extraction replicate (indicated by .R). For ease of comparison, taxa are coloured as per Supplementary Fig. 1B. Replicate pairs have similar taxonomic profiles. Fig. S3A. Genus-level taxonomic profile of CRC and non-CRC control samples. Each bar represents a sample labelled as follows: country of origin ( $\mathrm{AR}=$ Argentina; $\mathrm{CH}=\mathrm{Chile}$; IN = India; $\mathrm{VI}=$ Vietnam); disease status $(\mathrm{CRC}=\mathrm{CRC}$; NC= non-CRC control). For ease of comparison, taxa are coloured as per Supplementary Fig. 1B. South American samples generally have a high relative abundance of Bacteroides, and Asian samples a high relative abundance of Prevotella. Fig. S3B. The mean taxonomic composition (genus-level) of CRC and non-CRC control samples. Each bar represents the mean taxonomic composition of a group labelled as follows: country of origin $(A R=$ Argentina; $\mathrm{CH}=$ Chile; $\mathrm{IN}=$ India; $\mathrm{VI}=$ Vietnam); disease status (CRC = CRC; $N C=$ non-CRC control). For ease of comparison, taxa are coloured as per Supplementary Fig. 1B. South American samples have a high relative abundance of Bacteroides, and Asian samples a high relative abundance of Prevotella. Fig. S3C. LEfSe plot illustrating taxa enriched in South American (SA) compared with Asian (AS) samples. Fig. S4A. Distributions of relative abundance of genera of greatest importance to random forest models. The boxplots labelled 'All' are a summary of all of the studies, including the current study. The first 19 taxa are CRC-enriched (mean relative abundance) in the majority of studies; the final 5 taxa are controlenriched (mean relative abundance) in the majority of studies. Fig. S4B LEfSe plot illustrating taxa enriched in CRC compared with non-CRC controls for the current study cohort as a whole. Fig. S4C. LEfSe plot illustrating taxa enriched in CRC compared with non-CRC controls

(Argentina). Fig. S4D. LEfSe plot illustrating taxa enriched in CRC compared with non-CRC controls (Chile). Fig. S4E. LEfSe plot illustrating taxa enriched in CRC compared with non-CRC controls (India). Fig. S4F. LEfSe plot illustrating taxa enriched in CRC compared with non-CRC controls (Vietnam)

\section{Abbreviations}

AUC: Area under receiver operator characteristic curve; CRC: Colorectal cancer; DAC: Development Assistance Committee; EMP: Earth Microbiome Project; FTA: Flinders Technology Associates cards; gFOBT: Guaiac faecal occult blood test; LEfSe: Linear discriminant analysis Effect Size; LODO: Leaveone-dataset-out; PCoA: Principle coordinate analysis; RF: Random forest

\section{Acknowledgements}

Not applicable.

\section{Authors' contributions}

$C Y, H W$, RAS, and PQ: study design and supervision. RAS, PVN, CV, LCM, MB, MVD, TP, CTV, and JA: acquisition of data and samples. CY and AFB: sample processing. $C Y, H W, K T, Y Y$, and $C H$ : data analysis. $C Y, H W, K T, Y Y$, and $C H$ : drafting of the manuscript. CY, HW, RAS, PVN, CV, LCM, MB, MVD, TP, CTV, JA, $A F B, K T, Y Y, C H$, and PQ: critical revision of the manuscript. CY, RAS, and PQ: fundraising for the study. All authors read and approved the final version of the manuscript.

\section{Funding}

This work was funded by an Academy of Medical Sciences Global Challenges Research Fund Networking Grant (GCRFNG $\backslash 100433$ ) to PQ and RAS, a Wellcome Trust Clinical Research Training Fellowship (203524/Z/16/Z) to CY, a Pathological Society of Great Britain \& Ireland 'Visiting Fellowship' (2234) to CY and a Cancer Research UK Grand Challenge Initiative (Optimisticc

C10674/A27140) to PQ and CH. PQ is a National Institute of Health Research Senior Investigator. The funders had no role in study design, data collection, analysis, and interpretation, or in the writing of the report.

\section{Availability of data and materials}

The dataset (16S rRNA gene V4 amplicon data from faecal samples) supporting the conclusions of this article is available in the ENA repository [48]: https://www.ebi.ac.uk/ena/data/view/PRJEB36789

\section{Ethics approval and consent to participate}

Network members were granted local ethical approval for participant enrolment (Argentina: \#3507; Chile: CEC201828; India: IEC/2018/01, Indian Council of Medical Research: 2018-0337; Vietnam: QD.0604), and ethical approval for the whole study was granted by the University of Leeds (MREC17-077). Participants gave informed voluntary consent to take part in the study. The research conformed to the principles of the Helsinki Declaration.

\section{Consent for publication}

Not applicable.

\section{Competing interests}

The authors declare that they have no competing interests.

\section{Author details}

${ }^{1}$ Pathology \& Data Analytics, Leeds Institute of Medical Research at St James's University Hospital, University of Leeds, Level 4 Wellcome Trust Brenner Building, Leeds LS9 7TF, UK. ${ }^{2}$ Cancer Institute (WIA), Chennai, India. ${ }^{3}$ Can Tho University of Medicine and Pharmacy, Can Tho, Vietnam. ${ }^{4}$ Instituto de Medicina Traslacional e Ingeniería Biomédica (IMTIB) - CONICET - Instituto Universitario del Hospital Italiano (IUHI), Hospital Italiano de buenos Aires (HIBA), Buenos Aires, Argentina. ${ }^{5}$ Universidad de los Andes, Santiago, Chile. ${ }^{6}$ Department of Biostatistics, Harvard T.H. Chan School of Public Health, Harvard University, Boston, USA.

Received: 9 August 2020 Accepted: 4 February 2021

Published online: 16 February 2021

\section{References}

1. Bray F, Ferlay J, Soerjomataram I, Siegel RL, Torre LA, Jemal A. Global cancer statistics 2018: GLOBOCAN estimates of incidence and mortality worldwide for 36 cancers in 185 countries. CA: A Cancer J Clin. 2018;0(0):[no pagination]

2. Pleguezuelos-Manzano C, Puschhof J, Huber AR, van Hoeck A, Wood HM, Nomburg J, et al. Mutational signature in colorectal cancer caused by genotoxic pks+ E. coli. Nature. 2020;580(7802):269-73.

3. Allali I, Boukhatem N, Bouguenouch L, Hardi H, Boudouaya HA, Cadenas MB, et al. Gut microbiome of Moroccan colorectal cancer patients. Med Microbiol Immunol. 2018;207(3-4):211-25.

4. Alomair AO, Masoodi I, Alyamani EJ, Allehibi AA, Qutub AN, Alsayari KN, et al. Colonic mucosal microbiota in colorectal cancer: a single-center metagenomic study in Saudi Arabia. Gastroenterol Res Pract. 2018;2018: 5284754.

5. Faruk M, Ibrahim S, Adamu A, Rafindadi AH, Ukwenya Y, lliyasu Y, et al. An analysis of dietary fiber and fecal fiber components including $\mathrm{pH}$ in rural Africans with colorectal cancer. Intestinal research. 2018;16(1):99-108.

6. Bamola VD, Ghosh A, Kapardar RK, Lal B, Cheema S, Sarma P, et al. Gut microbial diversity in health and disease: experience of healthy Indian subjects, and colon carcinoma and inflammatory bowel disease patients. Microb Ecol Health Dis. 2017;28(1):1322447.

7. Loke MF, Chua EG, Gan HM, Thulasi K, Wanyiri JW, Thevambiga I, et al. Metabolomics and 16S rRNA sequencing of human colorectal cancers and adjacent mucosa. PLoS One. 2018;13(12):e0208584. 
8. Sonnenburg ED, Sonnenburg JL. The ancestral and industrialized gut microbiota and implications for human health. Nat Rev Microbiol. 2019; 17(6):383-90.

9. Vogtmann E, Chen J, Amir A, Shi J, Abnet CC, Nelson H, et al. Comparison of collection methods for fecal samples in microbiome studies. Am J Epidemiol. 2017;185(2):115-23.

10. Sinha R, Chen J, Amir A, Vogtmann E, Shi J, Inman KS, et al. Collecting fecal samples for microbiome analyses in epidemiology studies. Cancer Epidemiol Biomark Prev. 2016;25(2):407-16.

11. Dominianni C, Wu J, Hayes RB, Ahn J. Comparison of methods for fecal microbiome biospecimen collection. BMC Microbiol. 2014;14:103.

12. Wong WSW, Clemency N, Klein E, Provenzano M, lyer R, Niederhuber JE, et al. Collection of non-meconium stool on fecal occult blood cards is an effective method for fecal microbiota studies in infants. Microbiome. 2017; 5(1):114.

13. Taylor M, Wood HM, Halloran SP, Quirke P. Examining the potential use and long-term stability of guaiac faecal occult blood test cards for microbial DNA 165 rRNA sequencing. J Clin Pathol. 2017;70(7):600-6.

14. Byrd DA, Sinha R, Hoffman KL, Chen J, Hua X, Shi J, et al. Comparison of methods to collect fecal samples for microbiome studies using wholegenome shotgun metagenomic sequencing. mSphere. 2020;5(1):e00827-19.

15. von Huth $S$, Thingholm LB, Bang C, Rühlemann MC, Franke A, Holmskov U. Minor compositional alterations in faecal microbiota after five weeks and five months storage at room temperature on filter papers. Sci Rep. 2019; 9(1):19008.

16. Song SJ, Amir A, Metcalf JL, Amato KR, Xu ZZ, Humphrey G, et al. Preservation methods differ in fecal microbiome stability, affecting suitability for field studies. mSystems. 2016;1(3):[no pagination].

17. Vogtmann $E$, Chen J, Kibriya MG, Chen Y, Islam T, Eunes M, et al. Comparison of fecal collection methods for microbiota studies in Bangladesh. Appl Environ Microbiol. 2017;83(10):e00361-17.

18. Sierra MS, Forman D. Burden of colorectal cancer in Central and South America. Cancer Epidemiol. 2016;44:S74-81.

19. Vuong DA, Velasco-Garrido M, Lai TD, Busse R. Temporal trends of cancer incidence in Vietnam, 1993-2007. Asian Pac J Cancer Prev. 2010;11(3):739-45.

20. Pathy S, Lambert R, Sauvaget C, Sankaranarayanan R. The incidence and survival rates of colorectal cancer in India remain low compared with rising rates in East Asia. Dis Colon Rectum. 2012;55(8):900-6.

21. Chung RY, Tsoi KKF, Kyaw MH, Lui AR, Lai FTT, Sung JJ. A population-based age-period-cohort study of colorectal cancer incidence comparing Asia against the West. Cancer Epidemiol. 2019;59:29-36.

22. Ferlay J EM, Lam F, Colombet M, Mery L, Piñeros M, Znaor A Soerjomataram I and Bray F. Global Cancer Observatory: Cancer Today Lyon, France: International Agency for Research on Cancer. 2018 [Available from: https://gco.iarc.fr/today.

23. Gupta A, Dhakan DB, Maji A, Saxena R, P. K VP, Mahajan S, et al. Association of Flavonifractor plautii, a flavonoid-degrading bacterium, with the gut microbiome of colorectal cancer patients in India. mSystems. 2019;4(6): e00438-19.

24. Kostic AD, Gevers D, Pedamallu CS, Michaud M, Duke F, Earl AM, et al. Genomic analysis identifies association of Fusobacterium with colorectal carcinoma. Genome Res. 2012;22(2):292-8.

25. Earth Microbiome Project. [Available from: http://www.earthmicrobiome.org

26. Parada AE, Needham DM, Fuhrman JA. Every base matters: assessing small subunit rRNA primers for marine microbiomes with mock communities, time series and global field samples. Environ Microbiol. 2016;18(5):1403-14.

27. Apprill A, McNally S, Parsons RJ, Weber LK. Minor revision to V4 region SSU rRNA 806R gene primer greatly increases detection of SAR11 bacterioplankton. Aquat Microb Ecol. 2015;75(2):129-37.

28. Martin M. Cutadapt removes adapter sequences from high-throughput sequencing reads. EMBnetjournal. 2011;17(1):10-2.

29. Bolyen E, Rideout JR, Dillon MR, Bokulich NA, Abnet CC, Al-Ghalith GA, et al. Reproducible, interactive, scalable and extensible microbiome data science using QIIME 2. Nat Biotechnol. 2019;37(8):852-7.

30. Callahan BJ, McMurdie PJ, Rosen MJ, Han AW, Johnson AJA, Holmes SP. DADA2: high-resolution sample inference from Illumina amplicon data. Nat Methods. 2016;13:581.

31. Bokulich NA, Kaehler BD, Rideout JR, Dillon M, Bolyen E, Knight R, et al. Optimizing taxonomic classification of marker-gene amplicon sequences with QIIME 2's q2-feature-classifier plugin. Microbiome. 2018;6(1):90.
32. Camacho C, Coulouris G, Avagyan V, Ma N, Papadopoulos J, Bealer K, et al. BLAST+: architecture and applications. BMC Bioinformatics. 2009;10(1):421.

33. Quast C, Pruesse E, Yilmaz P, Gerken J, Schweer T, Yarza P, et al. The SILVA ribosomal RNA gene database project: improved data processing and webbased tools. Nucleic Acids Res. 2012;41(D1):D590-D6.

34. Shannon CE, Weaver W. The mathematical theory of communication: University of Illonois Press. Illonois: Champaign; 1949.

35. Kruskal WH, Wallis WA. Use of ranks in one-criterion variance analysis. J Am Stat Assoc. 1952;47(260):583-621.

36. Bray JR, Curtis JT. An ordination of the upland forest communities of southern Wisconsin. Ecol Monogr. 1957;27(4):325-49.

37. Oksanen J, Blanchet FG, Friendly M, Kindt R, Legendre P, McGlinn D, et al. vegan: Community Ecology Package. 2018. R package version 2.5-3. 2018 [Available from: https://CRAN.R-project.org/package=vegan.

38. Lloyd-Price J, Arze C, Ananthakrishnan AN, Schirmer M, Avila-Pacheco J, Poon TW, et al. Multi-omics of the gut microbial ecosystem in inflammatory bowel diseases. Nature. 2019;569(7758):655-62.

39. McMurdie PJ, Holmes S. phyloseq: an R package for reproducible interactive analysis and graphics of microbiome census data. PLOS ONE. 2013;8(4): e61217.

40. Breiman L. Random forests. Mach Learn. 2001;45(1):5-32.

41. Wiener ALaM. Classification and regression by randomForest. R News 2002; 2(3):18-22.

42. Robin X, Turck N, Hainard A, Tiberti N, Lisacek F, Sanchez J-C, et al. pROC: an open-source package for $\mathrm{R}$ and $\mathrm{S}+$ to analyze and compare ROC curves. BMC Bioinformatics. 2011;12(1):77.

43. Wirbel J, Pyl PT, Kartal E, Zych K, Kashani A, Milanese A, et al. Meta-analysis of fecal metagenomes reveals global microbial signatures that are specific for colorectal cancer. Nat Med. 2019;25(4):679-89.

44. Thomas AM, Manghi P, Asnicar F, Pasolli E, Armanini F, Zolfo M, et al. Metagenomic analysis of colorectal cancer datasets identifies cross-cohort microbial diagnostic signatures and a link with choline degradation. Nat Med. 2019;25(4):667-78.

45. Yachida S, Mizutani S, Shiroma H, Shiba S, Nakajima T, Sakamoto T, et al. Metagenomic and metabolomic analyses reveal distinct stage-specific phenotypes of the gut microbiota in colorectal cancer. Nat Med. 2019;25(6): 968-76

46. Segata N, Waldron L, Ballarini A, Narasimhan V, Jousson O, Huttenhower C. Metagenomic microbial community profiling using unique clade-specific marker genes. Nat Methods. 2012;9(8):811-4.

47. Segata N, Izard J, Waldron L, Gevers D, Miropolsky L, Garrett WS, et al. Metagenomic biomarker discovery and explanation. Genome Biol. 2011; 12(6):R60-R.

48. C Young, H Wood, R Seshadri, P Van Nang, C Vaccaro, L Contreras Melendez, et al. Data from the manuscript "The colorectal cancer-associated faecal microbiome of developing countries resembles that of developed countries": ENA repository; [Available from: https://www.ebi.ac.uk/ena/data/ view/PRJEB36789.

49. Ticinesi A, Nouvenne A, Corrente V, Tana C, Di Mario F, Meschi T. Diverticular disease: a gut microbiota perspective. J Gastrointestinal Liver Dis. 2019;28(3):327-37.

50. Escobar JS, Klotz B, Valdes BE, Agudelo GM. The gut microbiota of Colombians differs from that of Americans, Europeans and Asians. BMC Microbiol. 2014;14:311

51. Belforte FS, Fernandez N, Tonin Monzon F, Rosso AD, Quesada S, Cimolai $M C$, et al. Getting to know the gut microbial diversity of metropolitan Buenos Aires inhabitants. Front Microbiol. 2019;10:965.

52. Fujio-Vejar S, Vasquez $Y$, Morales $P$, Magne F, Vera-Wolf $P$, Ugalde JA, et al. The gut microbiota of healthy Chilean subjects reveals a high abundance of the phylum Verrucomicrobia. Front Microbiol. 2017;8:1221.

53. Dubey AK, Uppadhyaya N, Nilawe P, Chauhan N, Kumar S, Gupta UA, et al. LogMPIE, pan-India profiling of the human gut microbiome using 165 rRNA sequencing. Sci Data. 2018;5:180232.

54. Dhakan DB, Maji A, Sharma AK, Saxena R, Pulikkan J, Grace T, et al. The unique composition of Indian gut microbiome, gene catalogue, and associated fecal metabolome deciphered using multi-omics approaches. Gigascience. 2019;8(3): giz004.

55. Bhute S, Pande P, Shetty SA, Shelar R, Mane S, Kumbhare SV, et al. Molecular characterization and meta-analysis of gut microbial communities illustrate enrichment of Prevotella and Megasphaera in Indian subjects. Front Microbiol. 2016;7:660. 
56. Tandon D, Haque MM, R S, Shaikh S, P S, Dubey AK, et al. A snapshot of gut microbiota of an adult urban population from Western region of India. PLoS One. 2018;13(4):e0195643.

57. Dehingia M, Devi KT, Talukdar NC, Talukdar R, Reddy N, Mande SS, et al. Gut bacterial diversity of the tribes of India and comparison with the worldwide data. Scientific Reports. 2015;5:18563.

58. Carbonetto B, Fabbro MC, Sciara M, Seravalle A, Méjico G, Revale S, et al. Human microbiota of the Argentine population- a pilot study. Front Microbiol. 2016;7:51.

59. Dai Z, Coker OO, Nakatsu G, Wu WKK, Zhao L, Chen Z, et al. Multi-cohort analysis of colorectal cancer metagenome identified altered bacteria across populations and universal bacterial markers. Microbiome. 2018;6(1):70.

60. Brennan CA, Garrett WS. Fusobacterium nucleatum - symbiont, opportunist and oncobacterium. Nat Rev Microbiol. 2019;17(3):156-66.

61. Flemer B, Warren RD, Barrett MP, Cisek K, Das A, Jeffery IB, et al. The oral microbiota in colorectal cancer is distinctive and predictive. Gut. 2018;67(8): 1454-63.

62. Dejea CM, Wick EC, Hechenbleikner EM, White JR, Mark Welch JL, Rossetti $\mathrm{BJ}$, et al. Microbiota organization is a distinct feature of proximal colorectal cancers. Proc Natl Acad Sci U S A. 2014;111(51):18321-6.

63. Drewes JL, White JR, Dejea CM, Fathi P, Iyadorai T, Vadivelu J, et al. High-resolution bacterial 165 rRNA gene profile meta-analysis and biofilm status reveal common colorectal cancer consortia. NPJ Biofilms Microbiomes. 2017;3:34.

64. Tomkovich S, Dejea CM, Winglee K, Drewes JL, Chung L, Housseau F, et al. Human colon mucosal biofilms from healthy or colon cancer hosts are carcinogenic. J Clin Invest. 2019:130:1699-712.

65. Gupta VK, Paul S, Dutta C. Geography, ethnicity or subsistence-specific variations in human microbiome composition and diversity. Front Microbiol. 2017;8:1162

\section{Publisher's Note}

Springer Nature remains neutral with regard to jurisdictional claims in published maps and institutional affiliations.

Ready to submit your research? Choose BMC and benefit from:

- fast, convenient online submission

- thorough peer review by experienced researchers in your field

- rapid publication on acceptance

- support for research data, including large and complex data types

- gold Open Access which fosters wider collaboration and increased citations

- maximum visibility for your research: over $100 \mathrm{M}$ website views per year

At $\mathrm{BMC}$, research is always in progress.

Learn more biomedcentral.com/submissions 